\title{
A New Methodology for Aircraft HVDC Power Systems design
}

\author{
D. Hernández, M. Sautreuil, N. Retière, D. Riu \\ G2ELab - UMR 5269 \\ 38402 Saint-Martin-d'Hères Cedex - France \\ E-mail: (hernandez,sautreuil,retiere,riu)@g2elab.inpg.fr
}

\author{
O. Sename \\ Gipsa-Lab - UMR 5216 \\ 38402 Saint-Martin-d'Hères Cedex - France \\ E-mail: olivier.sename@gipsa-lab.inpg.fr
}

Abstract - A new methodology for aircraft HVDC power systems design is first proposed. The first goal is to design the network subsystems in order to obtain optimal devices from a weight point of view. The contribution of optimal linear multivariable control methods is shown to be major for this first design objective and $\mathrm{H}_{8}$ synthesis is applied in this paper. The second design objective is that the devices exhibit an adequate dynamical behavior when integrated into the DC network. But during design phases, the aircraft subsystems providen generally do not know the parameters of the DC network. To guarantee an easy integration of the subsystems, the device design should be robust to DC network uncertainties. Robust performance analysis method is applied in this paper and is integrated in the proposed methodology.

\section{INTRODUCTION}

The aircraft electric power system structures have been considerably changed for some years due to the development of efficient and reliable power electronics converters. This evolution has started by replacing more and more hydraulic devices by electric or hydro-electric devices in the "More Electrical" aircraft. This evolution will reach its final point when all the secondary power will be delivered through an electrical network. On-board aircraft electrical power system is then set to evolve, with the "All Electrical" concept, to a High Voltage DC structure, mainly for weight gain, efficiency, reliability and reduced maintenance cost reasons [1]. This evolution was initiated by the European aircraft manufacturers in the "Power Optimized Aircraft" EU project and is now followed by the "More Open Electrical Technologies" project $[2,3,4]$.

To achieve efficient operation conditions, several standards are imposed by the aircraft manufacturer to the individual devices suppliers. The goal is that, when assembled together, the whole DC system exhibits desired performances. These system level performances are defined by the aircraft manufacturer and are given in Fig. 1.

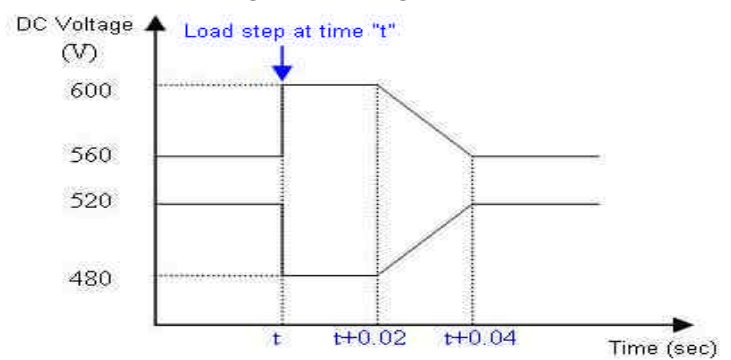

Fig. 1. Template for the DC voltage at the Point of Regulation (POR).
If these standards are not respected when the subsystems integration is carried out, some of the devices should be redesigned by their suppliers. This may lead to a long and costly design process. In this paper, the authors propose a new methodology for subsystem design in order to ease the integration phase while optimizing the weight of the subsystem. This methodology is described in Fig.2 and the focus is set on the contribution of optimal $\mathrm{H}_{8}$ control for this methodology.

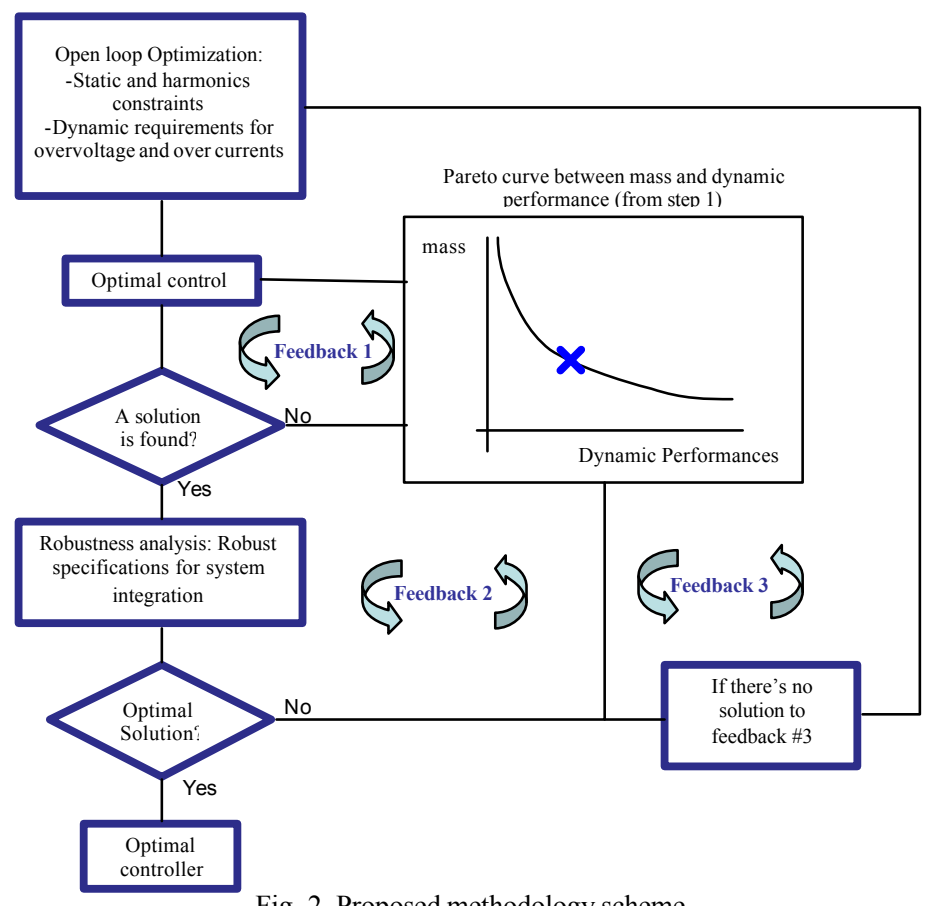

Fig. 2. Proposed methodology scheme.

The first step of the proposed design process is the optimization of the open-loop device using static and dynamic constraints. The static constraints are for example the harmonic standards imposed by the aircraft manufacturer. The dynamic constraints are the open loop over voltages and over currents. From this first step of the design process, a Pareto curve between required dynamic performances and the subsystem weight is obtained. For this, the authors propose to use the method described in [9] and [10]. From this open loop design, the second step is to find an optimal solution for the control. The authors propose to apply $H_{8}$ control for several reasons. First, this method is adapted for multivariable control, which means that several control 
objectives can be met at the same time using $H_{8}$ control. Second, it leads to optimal controllers: that means that if no solution to the control objectives is found, no solution exists. In this case, the designer should come back over the Pareto curve and take a less optimal weight point until the control objectives are can be met (feedback 1 in Fig. 2). At this step of the design process, an optimal close loop device has been designed from a weight point of view. But to ease the integration into the DC system, the authors suggested in [3] to impose to the device suppliers that the subsystems must be robust to DC side uncertainties. This is the third step of the design process. If the optimal controller of step 2 is robust from a performance point of view to these uncertainties, the optimal system has been found. If not, a less optimal weight point has to be considered until the robustness specifications are met (feedback 2 in Fig. 2). If a solution can not be found, the optimization has to be restarted from step 1 and a robustness analysis could be used to give new constraints for this first optimization (feedback 3 in Fig. 2).

This paper focuses on optimal control design using $H_{8}$ theory and robust performance analysis, which are two key points in the proposed methodology. In section II a brief theory review over the $H_{8}$ control is presented. The studied HVDC power channel is described and modeled in section III. The proposed application consists in a full wave rectifier feeding a load through a DC bus. This HVDC structure is detailed and an adequate dynamical model for $H_{8}$ control design is proposed. Section IV explains the design of a $H_{8}$ controller for the source described in section III. Finally, robust performance analysis is presented in section V. In this last section, the compromise between required performance for the device and its robustness is shown. These results could be used for feedback 3 (Fig. 2)

\section{MUltivariable OPTIMAL CONTROL}

\section{A. Generalities and principles}

The preliminary stage of the control design is generally to find and express the different relationships between the inputs and references of the system and the outputs to be controlled; in other words, to determinate the mathematical model of the system. In the case of a system with saturation phenomena or power electronics converters, a nonlinear model is obtained. Linearizing around an operating point yields to a linear model that is valid for small signal control design. The designer should then specify the control objectives, that is, the desired performances. Finally, the values of the controller are computed. Typical and conventional methods are based on the frequency domain analysis and use Bode, Black or Nyquist diagrams. They are graphical methods and they lead to very simple and fast control synthesis that, as a drawback, will be inevitably sub-optimal. The $H_{8}$ synthesis method presented in this work could be seen as a generalisation of these classic frequency methods, where the problem is formalized in a mathematical manner and the solution for the control of a multivariable system is calculated "automatically". The control synthesis using the optimal $H_{8}$ control theory minimizes the conception time, since it is a direct optimization of the control parameters. However, it is based on the use of numerical tools that could come out with convergence problems [5].

\section{B. $H_{8}$ Control Theory}

Multivariable synthesis techniques using $\mathrm{H}_{2}$ or $\mathrm{H}_{8}$ norms are some of the most important contributions of the modern robust control theory [6]. These techniques based on the computation of $\mathrm{H}_{2}$ or $\mathrm{H}_{8}$ norms, appeared after the first attempts of considering robustness objectives for control synthesis [7]. Being G(s) the transfer matrix between input vector and output vector, of dimension " $m$ " and " $p$ " respectively, the singular values is used for the gain notion. The singular value is given by [7]:

$\sigma_{i}(G(j \omega)):=\sqrt{\lambda_{i}\left(G(j \omega) G(-j \omega)^{T}\right)}=\sqrt{\lambda_{i}\left(G(-j \omega)^{T} G(j \omega)\right)}$

for $\mathrm{i}=1, \ldots, \min \{\mathrm{m}, \mathrm{p}\}$.

We will note afterwards $\bar{\sigma}(G)$ as the highest singular value and $\varrho(G)$ as the smallest. For a multivariable system, the gain at a given frequency will lie between the system's inferior and superior singular value [7].

The control problem of a given system could be reduced to the classic control configuration presented in Fig. 3.

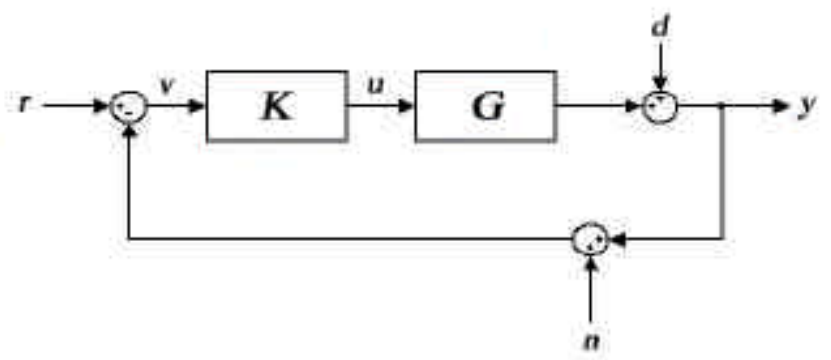

Fig. 3. Classic control configuration.

$K$ is the controller to be synthesized, " $r$ " are the system references, " $u$ " is the control law, "d" the system disturbances and "n", the system noise. From now on, we will consider that the system is not perturbed by noise signals $(n=0)$. The classic control scheme could be rewritten under the P-K form presented in Fig. 4.

So the optimal control design by means of the $H_{8}$ method consists in finding a stabilizing control such that, using the system output information, generates a control law $u$ that opposes the influence of $w$ and $z$ while minimizing the closed loop norm from $w$ to $z$ [5].

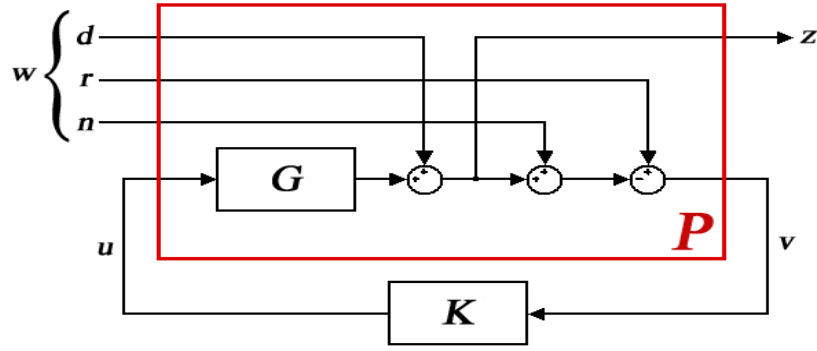

Fig. 4. Classic control configuration under the $\mathrm{P}-\mathrm{K}$ form. 
The system in Fig. 4 could be written as:

$$
\begin{aligned}
& {\left[\begin{array}{l}
z \\
v
\end{array}\right]=P(s)\left[\begin{array}{l}
w \\
u
\end{array}\right]=\left[\begin{array}{ll}
P_{11}(s) & P_{12}(s) \\
P_{21}(s) & P_{22}(s)
\end{array}\right]\left[\begin{array}{l}
w \\
u
\end{array}\right]} \\
& u=K(s) v
\end{aligned}
$$

We can find the transfer function between the input $w$ and the output $z$ using the Linear Fractional Transformation (LFT):

$$
\begin{aligned}
& z=F_{l}(P, K) w \\
& \quad \text { with: } F_{l}(P, K)=P_{11}+P_{12} K\left(I-P_{22} K\right)^{-1} P_{21}
\end{aligned}
$$

The optimal control problem is then reduced to find all stabilizing controllers for which the following expression is minimal [5]:

$\|\left. F_{l}(P, K)\right|_{\infty}=\max _{\omega} \sigma\left(F_{l}(P, K)(j \omega)\right)<\gamma$

where ? is a minimal boundary of the solution found by the optimization algorithm.

The mixed sensibility problem is usually solved to guarantee mult iple design objectives, for example disturbance and noise rejection. For a disturbance rejection design typically the $S / K S$ mixed sensitivity problem is solved. So finally, the problem is reduced to find a minimal boundary such that:

$\left\|\begin{array}{c}W_{1} S \\ W_{2} K S\end{array}\right\|_{\infty}<\gamma$

where $W_{1}$ and $W_{2}$ are frequency weights or weighting functions, which are defined to filter and to privilege some particular frequency domains. $S$ is the transfer from disturbance $d$ to output $z$, also called the sensitivity function.

\section{Modeling Uncertainties}

Uncertainties modeling is the preliminary step for robust performance analysis of the designed controller. Uncertainties are basically of two types. "Parametric uncertainties" represent the lack of knowledge on various parameters of the system (resistance, inductance, etc.). In our application these uncertainties are said to be "real". "Nonstructured uncertainties" represent the dynamics of the real system that were neglected in the mathematical model or the lack of knowledge of the model at high frequencies. These uncertainties are said to be "complex".

In this paper only parametric uncertainties on the DC filter are considered. For parametric uncertainties, the model structure is known but the $q_{p}$ parameters are uncertain. They are expressed by [3]:

$q_{p}=q_{\text {NOM }}\left(1+p_{q} \Delta\right)$

where $q_{N O M}$ is the nominal value of the parameter and:

$p_{q}=\left(q_{\max }-q_{\text {min }}\right) /\left(q_{\text {max }}+q_{\text {min }}\right)$

$|\Delta| \leq 1$

\section{D. $\mu$-Analysis}

The robust analysis methods are based upon the calculation of structured singular values $\mu[3]$. System uncertainties modeling is added to the general control configuration in Fig. 4. This same configuration can be rearranged in different configurations for the robust stability and robust performance analysis. These configurations are presented in Fig. 5.

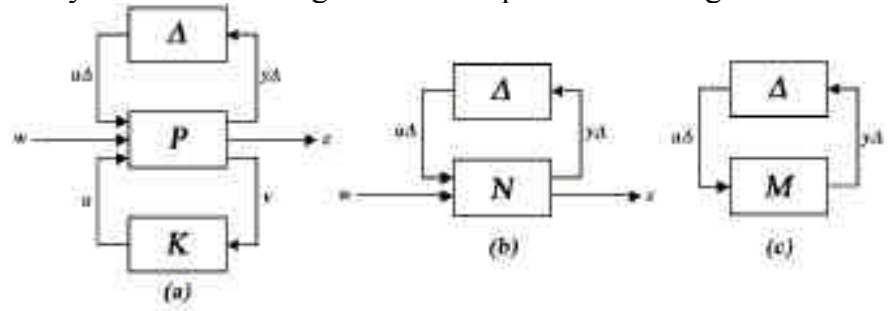

Fig. 5. (a) General control configuration with uncertainties. (b) Configuration for robust performance analysis. (c) Configuration for robust stability analysis.

The background for computing $\mu$ and its application to aircraft system robust performance analysis is presented in [3]. According to the different configurations (Fig. 5-b and Fig. 5-c), a resume of the several theorems for the robust analysis using $\mu$ computation could be as follows (Nominal Stability - NS, Nominal Performance - NP, Robust Stability RS and Robust Performance - RP):

$$
\begin{array}{ll}
N S \Leftrightarrow & \mathrm{N}(\mathrm{s}) \text { is internally stable } \\
N P \Leftrightarrow & \forall \omega \quad \mu_{\Delta f}\left(N_{22}(j \omega)\right)<1 \text { and NS } \\
R S \Leftrightarrow & \forall \omega \quad \mu_{\Delta i}\left(N_{11}(j \omega)\right)<1 \text { and NS } \\
R P \Leftrightarrow & \forall \omega \quad \mu_{\Delta}(N(j \omega))<1 \text { and NS }
\end{array}
$$

Tracing the structured singular values as a function of the frequency, and comparing with the previously described conditions, we can conclude over the uncertainties effects on the controller's stability and performance. For example, for robust stability, if an obtained value of $\mu$ at a given frequency is different to 1 , at this frequency, the model uncertainties could be tolerated $1 / \mu$ times, with the system stability always granted [5].

This is the so called $\boldsymbol{\mu}$-analysis, and a complete theory review could be found in 5]. Note that in practical cases, exact $\mu$ calculation is not possible and only upper and lower bounds are calculated.

\section{HVDC AIRCRAFT SYSTEM MODELING}

With the development of electricity as main energetic vector, the number of electric actuators increases. In "More Electric" aircrafts, the power distribution is mainly AC and $\mathrm{AC} / \mathrm{DC}$ followed by DC/AC conversion stages are needed to control motor loads. In "All Electric" aircrafts, the number of loads will increase so that to save weight and conversion stages, it becomes very interesting to distribute the electrical power with a common DC bus [1]. In these networks, the design of the DC source is a crucial point. Several solutions for the DC source structure can be studied. In [3], the authors studied a classical Autotransformer Rectifier Unit (ATRU) structure, for which the DC bus voltage is uncontrolled. In this paper, the DC bus voltage is directly controlled by a full 
wave controlled rectifier. The modeled HVDC structure is presented in Fig. 6.

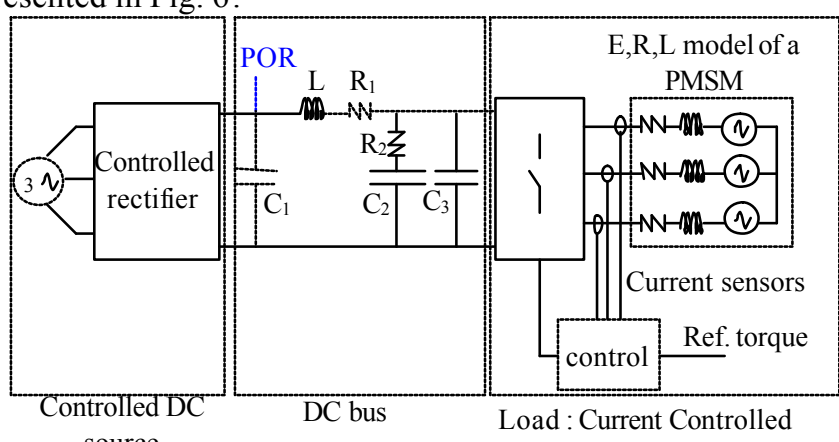

source

Fig. 6. Studied "All Electrical” HVDC aircraft structure.

The studied power channel is composed by an ideal three phase source in series with a (Rs,Ls) impedance, associated to a rectifier controlled by a control block $K$ (Fig. 7), a DC filter and a load, composed by a current-controlled inverter supplying a permanent magnet synchronous motor (PMSM). The detailed scheme of the DC source is presented in Fig. 7.

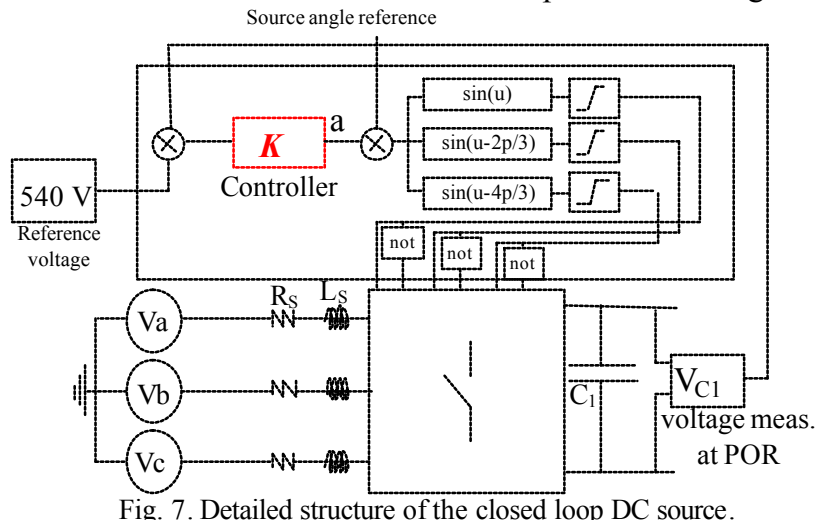

The parameters of the power channel and the system operating points are given in Table I.

TABLE I

MODEL PARAMETERS AND OPERATING POINTS

\begin{tabular}{|c|c|c|}
\hline Source parameters & $\begin{array}{c}\text { Rs } \\
\text { Ls } \\
\text { Va,Vb,Vc amplitude } \\
\text { Va,Vb,Vc frequency }\end{array}$ & $\begin{array}{c}5 \mathrm{mO} \\
66.6 \mu \mathrm{H} \\
325 \mathrm{~V} \\
400 \mathrm{~Hz} \\
\end{array}$ \\
\hline $\begin{array}{c}\text { DC bus } \\
\text { parameters }\end{array}$ & $\begin{array}{c}\mathrm{C}_{1} \\
\mathrm{~L} \\
\mathrm{R}_{1} \\
\mathrm{R}_{2} \\
\mathrm{C}_{2} \\
\mathrm{C}_{3} \\
\end{array}$ & $\begin{array}{c}680 \mu \mathrm{F} \\
1 \mathrm{mH} \\
10 \mathrm{mO} \\
1 \mathrm{O} \\
4700 \mu \mathrm{F} \\
630 \mu \mathrm{F} \\
\end{array}$ \\
\hline \multirow{5}{*}{$\begin{array}{l}\text { Operating points } \\
\text { of the system }\end{array}$} & $\mathrm{AC}$ source currents & $\begin{aligned} i_{\text {ded }} & =123.8 \mathrm{~A} \\
i_{\text {qeq }} & =111.9 \mathrm{~A}\end{aligned}$ \\
\hline & Rectifier control angle & $a_{e q}=0.0619$ \\
\hline & DC bus voltages & $\begin{array}{c}V_{C 1 e q}=540 \mathrm{~V} \\
V_{C 2 e q}=538.8 \mathrm{~V} \\
V_{C 3 e q}=538.8 \mathrm{~V}\end{array}$ \\
\hline & DC bus current & $i_{l e q}=111.34$ \\
\hline & Load Power & $P_{O}=60 \mathrm{~kW}$ \\
\hline
\end{tabular}

From electrical equations of the systems in $(\mathrm{d}, \mathrm{q})$ Concordia axes, the nonlinear average model of the open loop system is given by (14). The control angle is named $a$.

$\frac{d<i_{q}>_{0}}{d t}=-\frac{R_{s}}{L_{s}}<i_{q}>_{0}-\omega<i_{d}>_{0}+\frac{2 \sin \alpha<v_{C 1}>_{0}}{\pi L_{s}}+\frac{1}{L_{s}}<E_{q}>_{0}$ $\frac{d<i_{d}>_{0}}{d t}=\omega<i_{q}>_{0}-\frac{R_{s}}{L_{s}}<i_{d}>_{0}-\frac{2 \cos \alpha<v_{C 1}>_{0}}{\pi L_{s}}+\frac{1}{L_{s}}<E_{d}>_{0}$

$\frac{d<v_{C 1}>_{0}}{d t}=\frac{1}{C_{1}}\left(-\frac{3 \sin \alpha<i_{q}>_{0}}{\pi}+\frac{3 \cos \alpha<i_{d}>_{0}}{\pi}-<i_{L}>_{0}\right)$

$\frac{d<v_{C 2}>_{0}}{d t}=\frac{1}{R_{2} C_{2}}\left(<v_{C 3}>_{0}-<v_{C 2}>_{0}\right)$

$\frac{d<v_{C 3}>_{0}}{d t}=\frac{1}{C_{3}}\left(<i_{L}>_{0}-\frac{1}{R_{2}}\left(<v_{C 3}>_{0}-<v_{C 2}>_{0}\right)-\frac{P_{0}}{<v_{C 3}>_{0}}\right)$

$\frac{d<i_{L}>_{0}}{d t}=\frac{1}{L}\left(<v_{C 1}>_{0}-<v_{C 3}>_{0}-R_{1}<i_{L}>_{0}\right)$

The load was proven to exhibit a constant power load behavior in [3]. Its equation is given by: $i_{c h}=P_{0} / v_{C}, i_{c h}$ being the current absorbed by the load from the DC bus. The variables $i_{d}$ and $i_{q}$ correspond to the reference Park currents of the three phase source. $\left\langle i_{d}\right\rangle_{0}$ represents the average value of $\mathrm{i}_{\mathrm{d}}$, computed over the sliding period. Variables $\mathrm{V}_{\mathrm{C} 1}, \mathrm{~V}_{\mathrm{C} 2}, \mathrm{~V}_{\mathrm{C} 3}$ and $i$ corresponds to the state variables form capacitor voltages and inductor currents. These equations can be linearized and finally the following linear average model is obtained around an equilibrium point:

$\Delta \dot{x}=A \Delta x+B \Delta u$

$\Delta y=C \Delta x+D \Delta u$

With:

$\Delta x=\left[\begin{array}{llllll}\Delta i_{q} & \Delta i_{d} & \Delta V_{C 1} & \Delta V_{C 2} & \Delta V_{C 3} & \Delta i_{L}\end{array}\right]^{T} ; \Delta u=\left[\begin{array}{ll}\Delta \alpha & \Delta P_{0}\end{array}\right]^{T}$

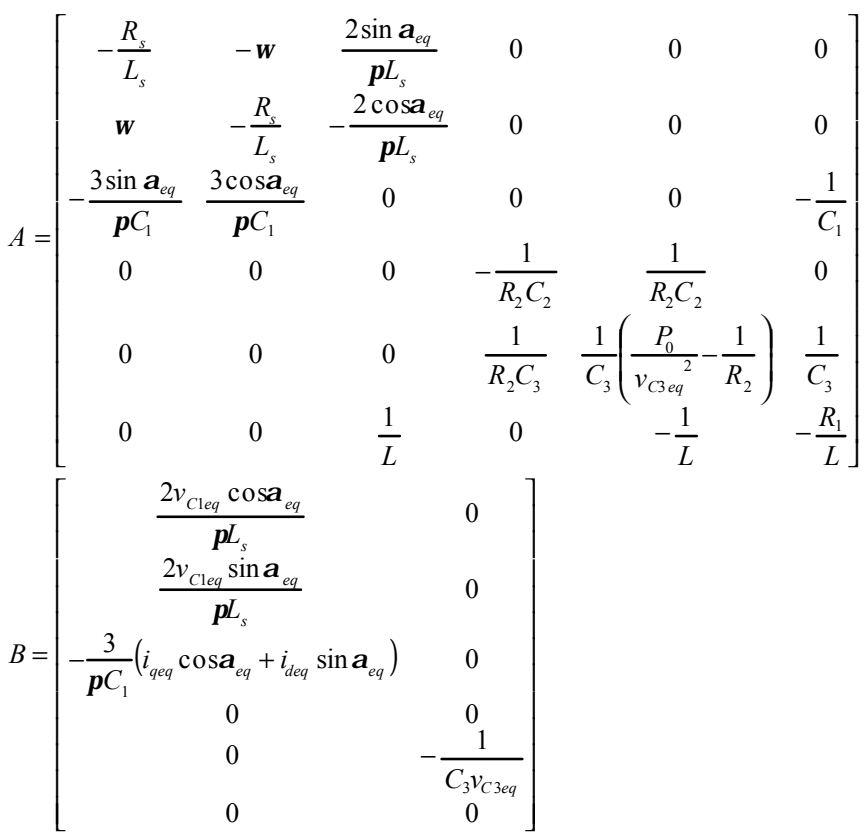

$C=\left[\begin{array}{llllll}0 & 0 & 1 & 0 & 0 & 0\end{array}\right]$ and $D=0$

The system inputs are:

- the control angle?a. 
- the load power ? $\mathrm{P}_{0}$, that corresponds to the system disturbance.

The system output is the POR voltage variation? $\mathrm{V}_{\mathrm{C} 1}$.

The transfer functions matrix of the system is given by:

$G(s)=\left[\begin{array}{l}G_{1}(s) \\ G_{2}(s)\end{array}\right]=\left[\begin{array}{l}\Delta V c_{1} / \Delta \alpha(s) \\ \Delta V c_{1} / \Delta P_{0}(s)\end{array}\right]$

\section{CONTROL DESIGN AND IMPLEMENTATION}

In the case of the system structure presented in Fig. 6, it can be demonstrated, by means of simple Bode diagrams, that the system structure has the natural energy necessary to reject the disturbance. Using a simplified mixed sensibility problem formulation, the control synthesis is carried out imposing a frequency domain weight only to the sensibility function. So the problem to be solved is reduced to:

$\left\|W_{\text {Perf }} S\right\|_{\infty}<\gamma$

$$
\text { with } S=\Delta V c_{1} / \Delta P_{0}
$$

A typical choice for the output performance weighting function $W_{\text {Perf }}$ is [7]:

$$
\frac{1}{W_{\text {Perf }}(s)}=\frac{s+\omega_{b} \varepsilon_{P}}{s / M_{S}+\omega_{b}}
$$

The weighting function parameters are chosen so that the performance requirements of Fig. 1 are respected. This requirement is defined for any load step value and then it is not possible to translate it exactly into small-signal requirements. In the time domain, the system performances can be assessed in terms of response time, static error, overshoot and damping. By limiting the maximal gain in the frequency domain, the damping of the time response is improved. The cut-off frequency in the frequency domain tunes the desired response time. This way, equivalencies between desired time domain performances and frequency domain specifications are made [3] and the respect of the small-signal specifications ensures that for large disturbances the specifications of Fig. 1 are met.

The result of the $H_{\infty}$ calculation leads to a $7^{\text {th }}$ order controller. The voltage at POR of the controlled system following a 50\% load step is given in Fig. 8. This result was carried out using the average nonlinear model.

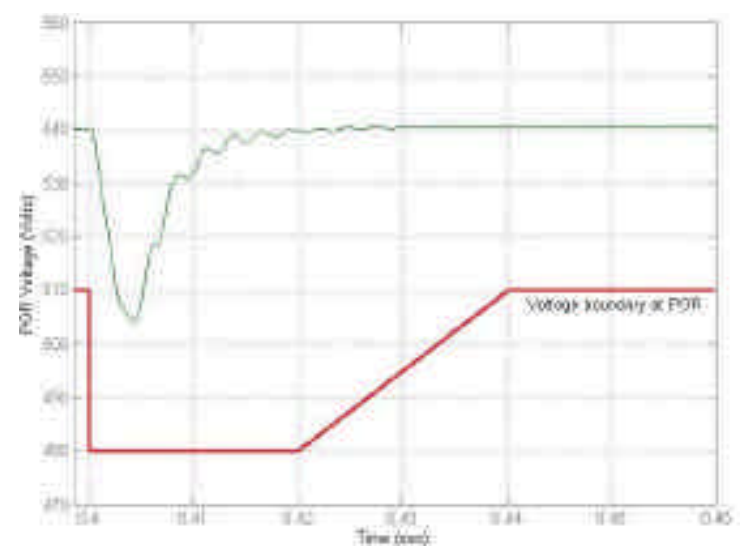

Fig. 8. Time response of the $H_{8}$ control.

It can be seen that the system response lies within the desired voltage boundary at the point of regulation, so it can be concluded that system performances are respected. In the optimization procedure presented in the introduction, if no solution was found, it would have been necessary to consider lower performance for the controller design, with for example higher $M_{s}$ (smaller damping) and smaller ? ${ }_{b}$ (lower time response) in (15). Section IV presented the $H_{\infty}$ control design. The robust performance of this controller is now analyzed in section $\mathrm{V}$.

\section{ROBUST PERFORMANCE ANALYSIS}

The robust performance of the $H_{8}$ control presented in section IV is analyzed using (13). The question is to know whether the closed loop system is robust (from a performance point of view) to the modelled parametric uncertainties or not. For this first analysis, the authors assume that the aircraft manufacturer imposed to the source supplier to be robust to $40 \%$ uncertainties on all the DC bus parameters in order to guarantee an easy integration of the device into the DC network. Robust performance analysis is a key point of the design approach proposed in the introduction of this paper.

The result for the $\mu$ upper and lower bound calculation of is shown in Fig. 9. For this analysis, the uncertainty level is $40 \%$ on all the parameters of the DC bus.

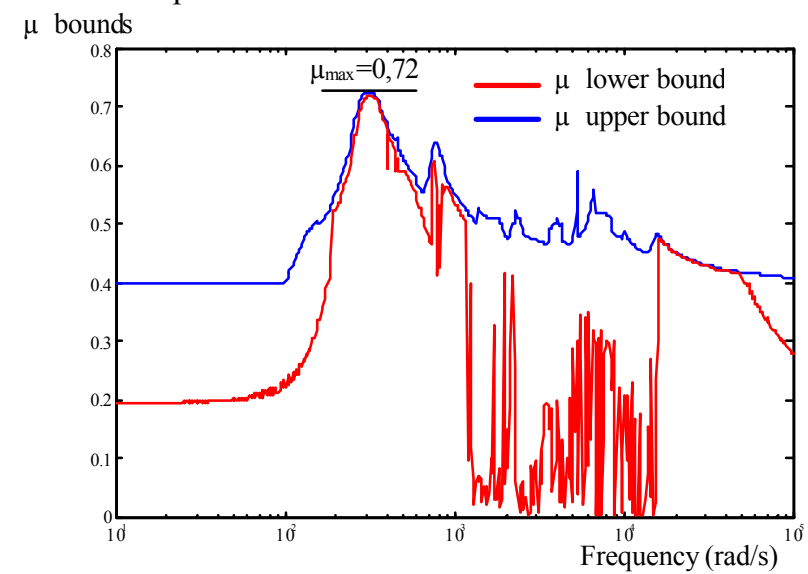

Fig. 9. Robust performance analysis for the $H_{8}$ control.

It can be seen that for $40 \%$ parametric uncertainties the system has robust performance, with a maximum $\mu$ value of $\mu_{\max }=0.72$. That means that for any combination of parameters in the parameter space defined by the $40 \%$ uncertainty set, the performance given by (18) is guaranteed. If the closed loop system was not found to be robust, the systems would not meet robustness specification given for system integration. It would have been necessary to come back over the performance requirements for the controller design as explained in the introduction. It was also explained that, in the case where no robust controller can be found, the optimization has to restart from step 1 (Fig. 2). For this, the robust performance analysis presented in Fig. 10 and Fig. 11 could help the designer to choose new specifications for the system dynamic performance at step 1 . 
The acceptable uncertainty size is now directly translated into a design margin, which means extended parameter combination possibilities when searching for an optimal solution from a weight point of view. Influence of time response requirements (cutoff frequency) and overshoot requirements over design margins are presented in Fig. 10 and Fig. 11 respectively. Fig. 10 shows how requiring a time response faster than $8 \mathrm{msec}$ (cutoff frequency of more than $5 * 24840 \mathrm{rad} / \mathrm{sec}$ ) leads to a drastic decrease in design margin and consequently a potential increase of the system weight. Fig. 11 shows again the robustness/performance compromise, while deteriorating response performances (overshoot) yields to more robust and lighter systems. As a conclusion, requiring very fast and damped disturbance rejection response may lead to a heavy design of the system. This clearly illustrates the compromise between system robustness and performance of the device design and makes Fig. 10 and Fig. 11 useful for the device design.

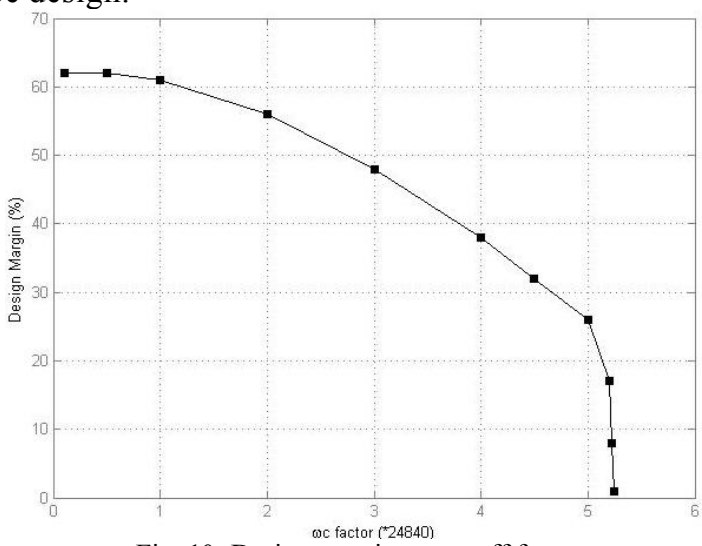

Fig. 10. Design margin vs. cutoff frequency.

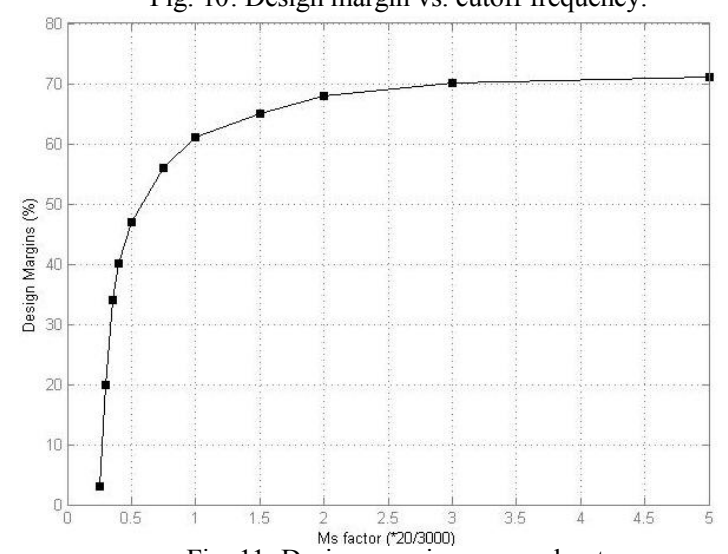

Fig. 11. Design margin vs. overshoot.

\section{CONCLUSION}

In this paper, a new methodology for subsystem design in aircraft DC power systems is proposed. Two key issues are underlined. The first one is the optimization of the devices from a weight point of view. The second one is the integration of the device in the DC power system. To ease the integration of the device, the authors propose to specify to the equipments suppliers to be robust to DC side uncertainties. But as shown in section $\mathrm{V}$, compromises have to be done between performance, robustness and system weight. This justifies the optimization procedure presented at the very beginning of this paper. Future publications will propose other optimal control design method than classical $\mathrm{H}_{8}$ control and will be compared together for the same power channel. The optimization approach proposed in this paper will also be fully applied to the design of an aircraft subsystem.

\section{ACKNOWLEDGMENT}

This research is being conducted in the frame of the MOET project, a FP6 European Integrated Project. The authors would like to thank MOET partners for model parameters and on board structure definition.

\section{REFERENCES}

[1] A. Emadi and M. Eshani. "Aircraft power systems: Technology, state of the art and future trends," IEEE AES Systems Magazine, 2000.

[2] F. Barruel, A. Caisley, N. Retière and J.L. Schanen. "Stability approach for vehicles DC power network: Aplication to aircraft on-board system," IEEE $36^{\text {th }}$ Power Electronics Spec. Conf., 2005.

[3] M. Sautreuil, N. Retière, D. Riu, O. Sename. "A generic method for robust performance analysis of aircraft DC power systems," $34^{\text {th }}$ Annual Conference of the IEEE Industrial Electronics Society IECON'08, 2008.

[4] More Open Electrical Technologies (MOET) project website (visited: June 2008): http://www.moetproject.eu

[5] S. Skogestad and I. Postlethwaite. "Multivariable feedback control, analysis and design," John Wiley \& Sons, 2005.

[6] P. Dorato. "A hist orical Review of Robust Control," IEEE Control Systems Magazine, 1987.

[7] G. Duc and S. Font. "Commande $H_{8}$ et $\mu$-analyse, des outils pour la robustesse," Hermes Science Publication, 1999. In french.

[8] P. Petitclair, S. Bacha and J. P. Rognon. "Averaged modelling and nonlinear control of an ASVC (Advanced Static VAR Compensator)," 27th Annual IEEE Power Electronics Specialists Conference, 1996.

[9] H. Nguyen-Huu, N. Retière, F. Wurtz, «Optimization of an electrical system using Pareto borders of each component. Application to an automotive drive chain ", IEEE-IECON, 2006.

[10] H. Nguyen Huu, B. Sareni , F. Wurtz, N. Retière, X. Roboam, «Comparison of self-adaptive evolutionary algorithms for multimodal optimization», accepted to present at Optimization and Inverse Problems in Electromagnetism Conf., 2008. 